\title{
Mechanisms of Neutrophil Accumulation in the Lungs of Patients with Idiopathic Pulmonary Fibrosis
}

\author{
Gary W. Hunninghake, James E. Gadek, Thomas J. Lawley, \\ and Ronald G. Crystal, Pulmonary Branch, National Heart, Lung, and Blood \\ Institute, Dermatology Branch, National Cancer Institute, \\ National Institutes of Health, Bethesda, Maryland 20205
}

A B S T R A C T Neutrophils are a characteristic feature of the alveolitis of idiopathic pulmonary fibrosis (IPF), a chronic disorder limited to lung. One mechanism by which neutrophils may be selectively attracted to lung and not other tissues is via the secretion of a neutrophil-specific chemotactic factor by alveolar macrophages. To evaluate the role of alveolar macrophages in modulating the migration of neutrophils to the lung in IPF, alveolar macrophages, obtained by bronchoalveolar lavage of patients with IPF, were evaluated for their ability to release a chemotactic factor for neutrophils. Unstimulated alveolar macrophages from normal individuals did not release the factor. In patients with IPF, there was a significant correlation between the proportions of neutrophils in lavage fluid and the release of a chemotactic factor for neutrophils by alveolar macrophages $(P<0.001)$. The chemotactic factor released by IPF alveolar macrophages was of low molecular weight (400-600), at least partially lipid in nature, and preferentially attracted neutrophils compared with monocytes. Several lines of evidence suggested that immune complexes in the lung stimulated alveolar macrophages of patients with IPF to release the chemotactic factor. First, immune complexes stimulated normal macrophages to release the factor. Second, there was a significant correlation between the release of the chemotactic factor by IPF alveolar macrophages and the levels of immune complexes in bronchoalveolar lavage fluid. Third, bronchoalveolar lavage fluid containing immune complexes stimulated normal macrophages to release the factor. Fourth, IPF alveolar macrophages that released large amounts of the chemotactic factor had an apparent suppression of their immunoglobulin (Ig)G Fc receptor function, sug-

This work was presented in part at the National Meeting of the American Federation for Clinical Research, May 1978, San Francisco, Calif.

Received for publication 6 May 1980 and in revised form 9 March 1981. gesting that immune complexes were bound to their surface. In contrast, the IgG Fc receptor function of IPF alveolar macrophages that released only small amounts of the factor was similar to that of normal macrophages. These studies suggest that neutrophils are attracted to the lung in patients with IPF by a potent chemotactic factor released by alveolar macrophages that have been stimulated, in vivo, via their IgG Fc receptor by immune complexes.

\section{INTRODUCTION}

Idiopathic pulmonary fibrosis (IPF) ${ }^{1}$ is a chronic disorder, limited to lung, characterized by the presence of an alveolitis, a disordering of lung parenchymal cells, and a disorganization of interstitial collagen (116). Current concepts of the pathogenesis of IPF suggests a central role for the alveolitis, since it precedes and likely mediates most of the parenchymal lesions that are found within the lung $(5,10-16)$.

The characteristic component of the alveolitis of IPF is the presence of neutrophils $(5,10-16)$. The presence of these cells within the lung in this disease has been documented by histological studies $(5,10$, 13) and by analysis of the inflammatory and immune effector cell populations obtained from bronchoalveolar lavage fluid $(5,10-16)$ and directly from open lung biopsies $(15,16)$. In contrast to the increased numbers of neutrophils in the IPF lung, the relative proportions of various other lung inflammatory and immune effector cell populations, including alveolar macrophages, T lymphocytes, and B lymphocytes, are normal (11-16). Thus, since neutrophils are potent mediators of inflammatory processes, it is likely that the neutrophil component of the alveolitis plays an important role in mediating parenchymal injury in these patients.

${ }^{1}$ Abbreviations used in this paper: hpf, high power field; IPF, idiopathic pulmonary fibrosis; ORBC, ox erythrocytes; PHA, phytohemagglutinin. 
Since neutrophils are rarely present in the lungs of normal individuals (11-16), and since IPF patients chronically have neutrophils within their lungs (1116), a mechanism must exist in the lungs of patients with IPF to attract neutrophils to the alveolar structures. One of the means by which neutrophils may be selectively attracted to the lung is by the release of a neutrophil-specific chemotactic factor by alveolar macrophages (17-23). Normal alveolar macrophages do not spontaneously release this chemotactic factor, but in vitro studies have demonstrated that normal macrophages may be stimulated to release the factor by a variety of agents including microorganisms, inorganic particulates, and immune complexes. Thus, if the release of this chemotactic factor by alveolar macrophages is relevant to the maintenance of the neutrophil alveolitis in IPF, then alveolar macrophages from individuals with this disorder should spontaneously release the chemotactic factor in vitro, presumably because the cells have been stimulated in vivo. The purpose of the present study, therefore, was twofold: first, to evaluate alveolar macrophages from patients with IPF for the spontaneous release of a chemotactic factor for neutrophils, and second, to determine the mechanism by which the IPF macrophages are stimulated to release the chemotactic factor.

\section{METHODS}

Study population: normal controls. The normal control population consisted of eight nonsmoking normal volunteers (age $31 \pm 5 \mathrm{yr}$; [all data are expressed as the mean \pm SEM] four males, four females). The pulmonary function tests and chest roentgenograms of all eight individuals were within normal limits and none was taking medication.

Study population: idiopathic pulmonary fibrosis. A diagnosis of idiopathic pulmonary fibrosis was made in 15 patients (age $51 \pm 7 \mathrm{yr}$; six females, nine males) by established criteria $(5,10-16)$, including open lung biopsy. All patients were in mid-course of their disease. Nine were taking no medication and six were taking prednisone $(15-20 \mathrm{mg}$, per os daily). Four of the patients had a history of cigarette smoking but they had discontinued smoking for at least $1 \mathrm{yr}$. The remaining patients were nonsmokers. As a group, the patients with IPF had the following functional abnormalities: vital capacity $68 \pm 12 \%$ predicted, total lung capacity $66 \pm 13 \%$ predicted, single breath diffusing capacity $55 \pm 9 \%$ predicted, forced expiratory volume in $1 \mathrm{~s} /$ forced vital capacity $98 \pm 6 \%$ predicted, resting $\mathrm{PaO}_{2} 75 \pm 9 \mathrm{~mm} \mathrm{Hg}$, and $\mathrm{PaO}_{2}$ at maximal steady-state exercise $54 \pm 13 \mathrm{~mm} \mathrm{Hg}$.

Bronchoalveolar lavage. Patients and normals were premedicated with valium $(2-10 \mathrm{mg}$, i.v. $)$ and atropine $(0.8 \mathrm{mg}$, i.m.). Following local anesthesia with $2 \%$ lidocaine spray, evaluation of the airways was carried out with a flexible fiberoptic bronchoscope (model BF-B2, Olympus Corporation of America, New Hyde Park, N. Y.). The tip of the bronchoscope was then wedged in B4 or B5 in the left lung. Lavage was carried out with a total of $100 \mathrm{ml}$ of sterile saline in five 20-ml aliquots with immediate vacuum aspiration after each aliquot. Immediately after lavage, the fluid was filtered through several layers of surgical gauze and the volume measured. The cells were then separated from the lavage fluid by centrifugation ( $500 \mathrm{~g}, 5 \mathrm{~min})$ and resuspended in Hanks' balanced salt solution (without $\mathrm{Ca}^{++}$or $\mathrm{Mg}^{++}$). The cells were then evaluated for total number (model FN, Coulter Electronics, Hialeah, Fla.), viability (trypan blue dye exclusion), and differential count (Wright-Giemsa stained cytocentrifuge preparation; Cytospin, Shandon Southern Instruments, Inc., Sewickley, Pa.). The bronchoalveolar lavage cells (in Hanks' balanced salt solution) were then subjected to Hypaque-Ficoll density centrifugation (24) to remove polymorphonuclear leukocytes, and the purified mononuclear cells (referred to subsequently as alveolar macrophages) were suspended in RPMI-1640 medium at a concentration of $10^{7}$ cells $/ \mathrm{ml}$.

Lavage fluid from the initial centrifugation was first concentrated to a volume of $1 \mathrm{ml}$ by pressure filtration using an Amicon appartus (Amicon EC-20, Amicon Corporation, Lexington, Mass.) and a UM2 membräne (molecular weight cutoff, 2,000$)$ as previously described $(7,12,25)$. Because protein concentrations in lavage fluid are dependent upon the degree of dilution of the epithelial lining fluid by the infused saline and upon the concentration of the fluid by membrane filtration, albumin was used as a standard for comparison of other protein components $(7,12,25)$. The concentrated lavage fluids were then diluted with normal saline so that each contained $0.5 \mathrm{mg}$ of albumin per $\mathrm{ml}$ of fluid. Immunoglobulin concentrations were measured by radial immunodiffusion.

Release of chemotactic activity by alveolar macrophages. To evaluate the release of chemotactic activity by alveolar macrophages, macrophages $\left(10^{6} \mathrm{cells} / \mathrm{ml}\right.$ in RPMI 1640 medium) recovered from Hypaque-Ficoll centrifugation were incubated for $3 \mathrm{~h}$ in sterile tissue culture plates (24 wells/ plate, 16-mm well Diam, Costar Data Packaging, Cambridge, Mass.) at $37^{\circ} \mathrm{C}$ in $5 \% \mathrm{CO}_{2}$ in air at $100 \%$ humidity. After $3 \mathrm{~h}$ in culture, the macrophage supernates were harvested by aspiration and filtered through $0.45-\mu \mathrm{M}$ filters (Swinnex-13, Millipore Corp., Bedford, Mass.) to remove the cells. All supernates were stored at $-20^{\circ} \mathrm{C}$ and were assayed within $2 \mathrm{wk}$ of harvesting.

The macrophage supernates were evaluated for chemotactic activity for neutrophils and mononuclear cells using a Blindwell micropore filter technique with nuclepore filters $(3-\mu \mathrm{m}$ pore size for neutrophils, $5-\mu \mathrm{m}$ pore size for mononuclear cells; Neuro Probe, Inc., Bethesda, Md.) (20). Mononuclear cells and neutrophils were obtained from peripheral blood of normal volunteers by sedimentation in Plasmagel (pyrogen free, HTI Corp., Buffalo, N. Y.) followed by Hypaque-Ficoll density centrifugation (24). The resulting mononuclear cell suspensions contained $>98 \%$ mononuclear cells of which $\sim 80 \%$ were lymphocytes and $20 \%$ were monocytes, while the neutrophil suspensions contained $>98 \%$ neutrophils. 200 $\mu \mathrm{l}$ of the responding cells [either neutrophils suspended at $2.3 \times 10^{6} \mathrm{cell} / \mathrm{s} / \mathrm{ml}$ in Gey's medium containing $2 \%$ bovine serum albumin, $100 \mathrm{U} / \mathrm{ml}$ penicillin, and $100 \mu \mathrm{g} / \mathrm{ml}$ streptomycin (all from Microbiological Associates, Walkersville, Md.) or peripheral blood mononuclear cells suspended at $3 \times 10^{6}$ cells $/ \mathrm{ml}$ in the same medium] was placed in the upper compartment of the chemotactic chamber and $150 \mu \mathrm{l}$ of the macrophage was placed in the lower compartment. Incubation times were $30 \mathrm{~min}$ for neutrophils and $90 \mathrm{~min}$ for mononuclear cells. The filters were stained with a Diff-Quick stain set (Harleco, American Hospital Supply Corp., Gibbstown, N. J.). Stained filters were examined at a magnification of 550 , and the number of cells that had migrated through the filters was counted in 5 to 10 random fields. Chemotactic activity was expressed as cells per high power field (hpf) reaching the lower surface of the filter in the presence of the 
macrophage supernates minus the cells/hpf reaching the lower surface of the filter in the presence of RPMI 1640 medium alone. Triplicate chambers were used for each experimental condition, and the means of the different experiments were compared using the two-tailed Student's $t$ test.

Partial characterization of the macrophage-derived chemotactic factor. Molecular sieve chromatography of the alveolar macrophage supernates was performed on a $2.5 \times 100-\mathrm{cm}$ column of Sephadex G-25 (Pharmacia Fine Chemicals, Piscataway, N. J.). The column was packed and eluted with $0.85 \% \mathrm{NaCl}-40 \mathrm{mM}$ phosphate, $\mathrm{pH} 7.4$ (phosphate-buffered saline) at $4^{\circ} \mathrm{C}$. 3-ml fractions were collected and assayed directly for chemotactic activity using the nuclepore filter assay. The G-25 Sephadex column was calibrated with cytochrome $c\left(12,500\right.$ daltons), vitamin $\mathrm{B}_{12}$ (1,350 daltons), and $\left[{ }^{3} \mathrm{H}\right]$ thymidine (242 daltons).

The solubility characteristics of the chemotactic factor released by alveolar macrophages were evaluated by extracting $5 \mathrm{ml}$ of the macrophage supernates twice with equal volumes of ethyl acetate, ether, or hexane (20). After each extraction, the organic solvents were dried with a stream of $\mathrm{N}_{2}$ and the extracted material resuspended in $5 \mathrm{ml}$ of RPMI 1640. This material, as well as the remaining aqueous phases, were then tested for chemotaxis for neutrophils as described above. Prior to testing, the aqueous phases were bubbled with $\mathrm{N}_{2}$ to remove any residual organic solvent. In all cases, $>90 \%$ of the chemotactic activity of the original macrophage supernates was recovered in the organic plus aqueous phases.

Evaluation of lavage fluid for stimuli that induce macrophages to secrete the chemotactic factor. To determine whether stimuli were present in lavage fluid that were capable of inducing alveolar macrophages to release the chemotactic factor, macrophages from normal nonsmoking individuals were incubated either alone or in the presence of lavage fluid (concentrated to contain $0.5 \mathrm{mg}$ albumin $/ \mathrm{ml}$ ). The macrophage cultures were prepared by mixing $0.7 \mathrm{ml}$ RPMI 1640, $0.1 \mathrm{ml}$ of a suspension of alveolar macrophages $\left(10^{7}\right.$ cells $\left./ \mathrm{ml}\right)$, and $0.2 \mathrm{ml}$ of lavage fluid. After $1 \mathrm{~h}$ of incubation with the lavage fluid, the original media was removed by aspiration and replaced with $1 \mathrm{ml}$ of fresh media (RPMI 1640). Following an additional $3 \mathrm{~h}$ in culture the macrophage supernates were harvested, stored, and evaluated for chemotactic activity as described above. As a control, the lavage fluid itself was also tested for chemotactic activity.

Detection of immune complexes in serum and lavage fluid. Serum and lavage fluid were evaluated for the presence of immune complexes using a ${ }^{125} \mathrm{I}-\mathrm{Cl}$-binding assay as previously described $(26,27)$. Briefly, $0.2 \mathrm{ml}$ of serum or concentrated lavage fluid (containing $0.5 \mathrm{mg}$ albumin) was incubated with $0.4 \mathrm{ml}$ of $\mathrm{Na}_{2}$ EDTA $(0.2 \mathrm{M}, \mathrm{pH} 7.5)$ for 30 min at $37^{\circ} \mathrm{C}$. After addition of $200 \mathrm{ng}$ of ${ }^{125} \mathrm{I}-\mathrm{Clq}, 1 \mathrm{ml}$ of $4 \%$ (wt/vol) polyethylene glycol (Carbowax, 6,000, mol wt, Fisher Scientific Co., Pittsburgh, Pa.) was added to the mixture to make a concentration of $2.5 \%$ polyethylene glycol. The mixture was incubated on ice for $1 \mathrm{~h}$ and then centrifuged at $1,000 \mathrm{~g}$ for $20 \mathrm{~min}$. The supernate was decanted and the residual radioactivity was counted without washing the pellet. Results were expressed as percentage of total precipitable protein bound ${ }^{125}$ I present (determined by $10 \%$ trichloroacetic acid precipitation). All samples were tested in duplicate. Normal human serum or lavage fluid was used as a standard in each assay. Positive controls consisted of serial dilutions of heat-aggregated human gamma globulin (Miles Laboratories, Inc., Elkhart, Ind.) added to the standard normal human serum or the standard normal lavage fluid. The least amount of aggregated gamma globulin that could be detected with the assay was $5 \mu \mathrm{g}$. The Clq binding activity of $5 \mu \mathrm{g}$ of aggregated gamma globulin is $6.2 \% ; 10 \mu \mathrm{g}=14.7 \% ; 25$ $\mu \mathrm{g}=36.8 \% ; 50 \mu \mathrm{g}=53.8 \% ;$ and $100 \mu \mathrm{g}=74.9 \%$. The $\mathrm{Cl}_{\mathrm{q}}$ binding activity of serum from all normal individuals is $<10 \%$ and the $\mathrm{Clq}$ binding activity of lavage fluid from all normal individuals is less than the sensitivity of the assay (i.e., $<6.2 \%$ ). The test sera and lavage fluids were stored at $-40^{\circ} \mathrm{C}$.

To determine whether immune complexes might be present in the cytoplasm of alveolar macrophages as well as in serum and lavage fluid, cytocentrifuge preparations of macrophages were fixed in glutaraldehyde ( $50 \%$ aqueous solution; Fisher Scientific Co.) and then exposed to an appropriate dilution of a fluorescein conjugated $F\left(a^{\prime}\right)_{2}$ goat anti-human IgG ( $\gamma$-chain specific; N. L. Cappel Laboratories, Inc., Cochranville, $\mathrm{Pa}$.) reagent. The macrophages were then washed and examined for intracytoplasmic fluorescence as previously described (11).

Evaluation of alveolar macrophage Fc-receptor function.

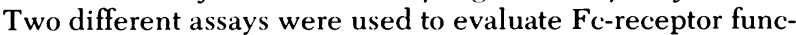
tion of alveolar macrophages: (a) release of chemotactic factor by alveolar macrophages following stimulation in vitro with IgG-coated ox erythrocytes (ORBC) (20); and (b) killing of IgG-coated ORBC by alveolar macrophages (antibodydependent cellular cytotoxicity) (28-30).

To evaluate the ability of IgG-coated ORBC to stimulate the release of chemotactic activity by alveolar macrophages, the macrophages were incubated either alone or in the presence of IgG-coated ORBC. In further experiments Sepharose$4 B$ was added to the cultures. Sepharose-4B was used as an additional stimulus since this agent also stimulates alveolar macrophages to release chemotactic factor, but not via the macrophage IgG-Fc receptor (20). The IgG-coated ORBC were prepared by coating the ORBC with subagglutinating amounts of rabbit IgG anti-ORBC antibody (N. L. Cappel Laboratories, Inc.) and suspending the cells in RPMI 1640 at a concentration of $5 \times 10^{8}$ cell s/ml. Sepharose- $4 \mathrm{~B}$ particles (Pharmacia Fine Chemicals) were prepared by washing the particles three times in Hanks' balanced salt solution and resuspending them in RPMI 1640 at a concentration of $10 \%$ by volume. The macrophage cultures were prepared by mixing $0.8 \mathrm{ml}$ of RPMI 1640, $0.1 \mathrm{ml}$ of a suspension of alveolar marcophages $\left(10^{7}\right.$ cells $\left./ \mathrm{ml}\right)$, and $0.1 \mathrm{ml}$ of the stimuli (IgGcoated ORBC and for Sepharose-4B). After $1 \mathrm{~h}$ of incubation, the original media was removed by aspiration and replaced with $1 \mathrm{ml}$ of fresh media (RPMI 1640). Following an additional $3 \mathrm{~h}$ in culture, the macrophage supernates were harvested and stored as described above. Since corticosteroids are known to inhibit $\mathrm{Fc}$ receptor function of alveolar macrophages, only alveolar macrophages from untreated patients were evaluated in this assay (28-29).

To evaluate the capacity of alveolar macrophages to mediate antibody dependent cellular cytotoxicity, the macrophages were co-cultured with ${ }^{51} \mathrm{Cr}$ labeled-ORBC coated with either IgG-anti-ORBC antibody or with phytohemagglutinin (PHA; Wellcome Research Laboratory, Beckenham, England) (28-30). PHA-coated ORBC were used as additional targets since killing of these cells is mediated by alveolar macrophages, but not via the macrophage IgG-Fc receptor $(28,29)$. IgG-coated ORBC were prepared as described above. PHA-coated ORBC were prepared by incubating ORBC with $10 \mu \mathrm{g} / \mathrm{ml}$ of PHA for $30 \mathrm{~min}$ at $37^{\circ} \mathrm{C}$. The cells were then washed and resuspended in RPMI 1640 at a concentration of $5 \times 10^{8}$ cells $/ \mathrm{ml}$. Both IgG-coated-ORBC and PHA-coated ORBC were labeled with ${ }^{51} \mathrm{Cr}$ as previously described (23-29). Alveolar macrophages were co-cultured with the ORBC targets using a previously described cytotoxicity assay $(31,32)$. Briefly, cultures were performed in $1 \times 7.5-\mathrm{cm}$ plastic tubes containing a total volume of $1 \mathrm{ml}$ RPMI 1640 with $10 \%$ fetal calf serum. In each assay ${ }^{51} \mathrm{Cr}$-labeled ORBC coated 
with either IgG or PHA ( $10^{5}$ cells) were incubated for $4 \mathrm{~h}$ with macrophage at various effector to target cell ratios. ${ }^{51} \mathrm{Cr}$ release from the target cells into the supernate was determined and the percentage of cytotoxicity calculated as previously described (28-32). Only alveolar macrophages from untreated patients were evaluated in this assay.

\section{RESULTS}

Bronchoalveolar lavage was performed without difficulty in each of the normal individuals and patients with IPF. Visualization of the tracheobronchial tree prior to lavage demonstrated normal airways in all individuals, with no evidence of inflammatory airway disease. There was no significant difference in the total fluid recovered from the normals and patients $(P>0.2)$. The recovered fluid was sterile in all cases, except for rare oral flora.

Presence of neutrophils in lung of normal individuals and patients with IPF. The cell yield per standard $100 \mathrm{ml}$ bronchoalveolar lavage was $10 \pm 3 \times 10^{6}$ cells in normals and $18 \pm 5 \times 10^{6}$ cells in patients with IPF. In each of the eight normals, $<1 \%$ of the cells that were present in lavage fluid were neutrophils (Fig. 1); the remainder of the bronchoalveolar cells were alveolar macrophages $(94 \pm 3 \%)$ and lymphocytes $(6 \pm 1 \%)$. In marked contrast to normals, significant numbers of neutrophils were present in lavage fluid of most of the patients with IPF (Fig. 1). In 9 of the 15 patients, neutrophils comprised $>10 \%$ of the bronchoalveolar cells. Three of the patients with $>10 \%$ neutrophils in lavage fluid and three patients with $<10 \%$ neutrophils in lavage fluid were receiving corticosteroids at the time of the study; the remaining patients in each group were not receiving medication. In patients with $>10 \%$ neutrophils in lavage fluid, there was no significant difference in the proportions of neutrophils between ex-smokers and nonsmokers $(P>0.2)$.

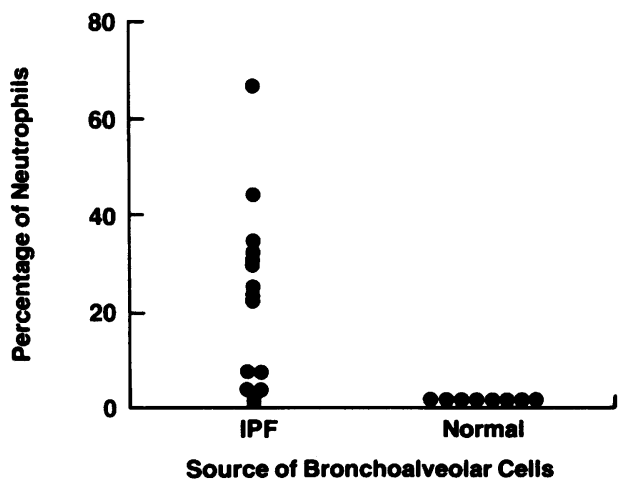

Figure 1 Percentage of neutrophils in bronchoalveolar lavage fluid of normal individuals and patients with IPF. The percentage of neutrophils is on the ordinate and the source of the cells is on the abscissa.
Release of a chemotactic factor for neutrophils by alveolar macrophages from normals and patients with IPF. Alveolar macrophages of normal individuals did not release detectable amounts of a chemotactic factor for neutrophils following $3 \mathrm{~h}$ in culture (Fig. 2). In contrast, supernates of alveolar macrophages from 10 of the 15 patients with IPF contained chemotactic activity for neutrophils. In patients with IPF, there was a strong correlation between the proportion of neutrophils in lavage fluid and the release of a neutrophil chemotactic factor by alveolar macrophages $(P<0.001)$. In five of the six patients with $<10 \%$ neutrophils in lavage fluid, chemotactic activity for neutrophils in alveolar macrophage supernates was either not detected or was present at very low levels; only one patient had sufficient chemotactic activity in the undiluted macrophage supernate to re-

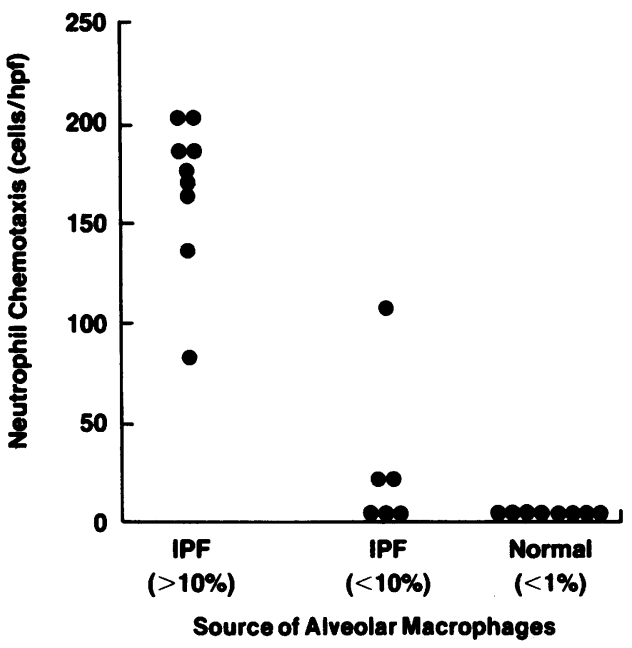

Figure 2 Release of a chemotactic factor for neutrophils by alveolar macrophages from normal individuals and patients with IPF. Following recovery from the patient, the macrophages were cultured for $3 \mathrm{~h}$. The chemotactic activity of the macrophage supernates is on the ordinate and the source of the macrophages is on the abscissa. "IPF $(>10 \%)$ " = alveolar macrophages from IPF patients with $>10 \%$ neutrophils in bronchoalveolar lavage fluid; "IPF $(<10 \%)$ " = alveolar macrophages from IPF patients with $<10 \%$ neutrophils in bronchoalveolar lavage fluid; "normal $(<1 \%)$ " = alveolar macrophages from normal individuals; all of these individuals had $<1 \%$ neutrophils in bronchoalveolar lavage fluid. No significant amounts of chemotactic activity were detected in macrophage supernates from normal individuals and five of the six IPF $(<10 \%)$ patients. An undiluted macrophage supernate from one IPF $(<10 \%)$ patient had sufficient chemotactic activity to result in a $50 \%$ maximal migration of neutrophils in the chemotaxis assay $\left(\mathrm{ED}_{50}\right)$. In four of the nine IPF $(>10 \%)$ patients, sufficient chemotactic activity was present at a 1:10 dilution of the macrophage supernates to result in a $50 \%$ effective dose $\left(E D_{50}\right)$ migration of neutrophils; in the other five IPF $(>10 \%)$ patients, sufficient activity was present in undiluted macrophage supernates to result in an $E D_{50}$ migration of neutrophils. 
sult in a 50\% maximal migration of neutrophils in the chemotaxis assay (20). By comparison, alveolar macrophage supernates from all patients with $>10 \%$ neutrophils in lavage fluid contained large amounts of chemotactic activity for neutrophils. In four of these nine patients, sufficient chemotactic activity was present in a 1:10 dilution of the supernates to result in at least a $50 \%$ maximal migration of neutrophils in the chemotaxis assay. In the other five patients, sufficient chemotactic activity was present in undiluted macrophage supernates to result in at least a $50 \%$ maximal migration of neutrophils.

The chemotactic factor released by alveolar macrophages from patients with IPF was of low molecular weight (400-600 mol wt) and had more chemotactic activity for peripheral blood neutrophils than for peripheral blood monocytes (Fig. 3). In addition, the chemotactic factor was also at least partially lipid in nature (Table I). $85 \%$ of the activity of the chemotactic factor was removed from the macrophage supernates following extraction into ethyl acetate, $70 \%$ with ether, and $40 \%$ with hexane. In each case, the activity that was removed from the macrophage supernates was completely recovered from the respective organic solvents.

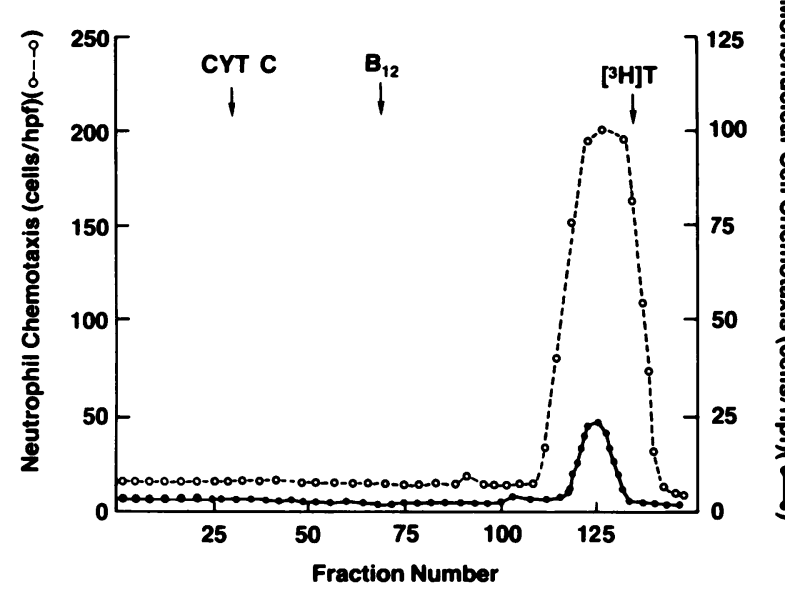

Figure 3 Sephadex G-25 column chromatography of the chemotactic factor present in supernates of alveolar macrophages from patients with idiopathic pulmonary fibrosis. The column was eluted at $4^{\circ} \mathrm{C}$ with $40 \mathrm{mM}$ phosphatebuffered saline, $\mathrm{pH}$ 7.4. The chemotactic activity of the macrophage supernates (expressed as cells migrated per hpf) for neutrophils is on the ordinate at the left and the chemotactic activity for mononuclear cells is on the ordinate at the right. Molecular weight markers include: cytochrome $c$ (CYT C; $12,500 \mathrm{~mol} \mathrm{wt}$ ), vitamin $\mathrm{B}_{12}(1,350 \mathrm{~mol} \mathrm{wt})$, and $\left[{ }^{3} \mathrm{H}\right]$ thymidine $\left(\left[{ }^{3} \mathrm{H}\right] \mathrm{T} ; 242 \mathrm{~mol} \mathrm{wt}\right)$. Fractions with the greatest amount of chemotactic activity could be diluted fivefold and still result in a $50 \%$ maximal migration of neutrophils in the chemotaxis assay. In contrast, these same fractions, when undiluted, did not result in a 50\% maximal migration of monocytes in the chemotaxis assay.
TABLE I

Organic Solvent Extraction of the Alveolar Macrophage Chemotactic Factor Derived from Patients with Idiopathic Pulmonary Fibrosis

\begin{tabular}{lc}
\hline \multicolumn{1}{c}{ Solvent } & $\begin{array}{c}\text { Percent original activity } \\
\text { extracted }\end{array}$ \\
\hline Chemotactic factor alone & 100 \\
Extracted into ethyl acetate & 85 \\
Extracted into ether & 70 \\
Extracted into hexane & 40 \\
\hline
\end{tabular}

Alveolar macrophages $\left(10^{6} \mathrm{cells} / \mathrm{ml}\right)$ were incubated for $3 \mathrm{~h}$ in RPMI 1640 medium. $5 \mathrm{ml}$ of the macrophage supernates were then extracted twice with equal volumes of ethyl acetate, ether, or hexane. Following extraction, the organic solvents were dried with a stream of $\mathrm{N}_{2}$ and the extracted material resuspended in $5 \mathrm{ml}$ of RPMI 1640. This material, as well as the remaining aqueous phases, were then tested for chemotaxis for neutrophils. In all cases, $>90 \%$ of the chemotactic activity of the original macrophage supernates was recovered in the organic plus aqueous phases. The percentage of original activity was estimated by determining the dilution of the chemotactic factor that resulted in a $50 \%$ maximal migration of neutrophils in the chemotaxis assay as previously described (20).

Presence of immune complexes in blood of normals and patients with IPF. The release of the chemotactic factor by alveolar macrophages correlated with the presence of circulating immune complexes in patients with IPF (Fig. 4). In normal individuals, alveolar macrophages did not release the chemotactic factor and the serum $\mathrm{Clq}$ binding activity was $<10 \%$. The serum $\mathrm{Clq}$ binding activity was also $<10 \%$ in patients with IPF whose alveolar macrophages did not release the chemotactic factor or released only small amounts of the factor (Fig. 4). In marked contrast, there was a significant elevation in the serum Clq binding activity in patients with IPF whose alveolar macrophages released large amounts of the chemotactic factor compared to normals and compared to patients with IPF whose macrophages did not release large amounts of the factor $(P<0.01$ both comparisons, Fig. 4).

Presence of immune complexes in lung of normals and patients with IPF. Several lines of evidence suggested that immune complexes were present in lung as well as in blood of IPF patients whose macrophages released the chemotactic factor.

First, the Clq binding activity was significantly increased in lavage fluid of patients with IPF whose alveolar macrophages released the chemotactic factor compared to normals and compared to patients with IPF whose macrophages did not release the factor (Table II; $\boldsymbol{P}<0.01$ each comparison).

Second, lavage fluid obtained from IPF patients with circulating immune complexes stimulated macrophages from normal individuals to release the chemotactic 


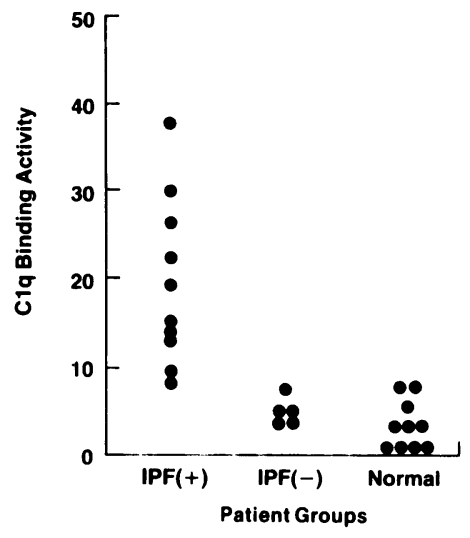

FIGURE $4{ }^{125} \mathrm{I}-\mathrm{Clq}$ binding activity in serum of normal individuals and patients with IPF. The ${ }^{125} \mathrm{I}-\mathrm{Cl}$ q binding activity is on the ordinate and the patient groups are indicated on the abscissa. "IPF (+)" - IPF patients whose alveolar macrophages release large amounts of a chemotactic factor for neutrophils (>50 cells/hpf, see Fig. 2); "IPF(-)" = IPF patients whose alveolar macrophages release either no detectable amounts of chemotactic factor or only small amounts of the factor ( $<50$ cells/hpf, see Fig. 2); "normal" = normal individuals.

factor (Fig. 5). In contrast, lavage fluid from normals and patients with IPF without circulating immune complexes did not stimulate normal macrophages to release

TABLE II

${ }^{125}$ I-C1q Binding Activity in Bronchoalveolar Lavage Fluid of Normals and Patients with Idiopathic Pulmonary Fibrosis

\begin{tabular}{cc}
\hline Patient group & Clq binding activity in lavage fluid \\
\hline & Percent protein bound ${ }^{125} I^{*}$ \\
Normals & Not detected $\$$ \\
IPF $(+) \S$ & $11.2 \pm 1.2^{11}$ \\
IPF $(-)$ & Not detected $\$$ \\
\hline
\end{tabular}

* Clq binding activity was measured using an ${ }^{125} \mathrm{I}$-Clq-binding assay $(26,27)$.

$\$$ The Clq binding activity of lavage fluid from all normal individuals and IPF - patients was less than the sensitivity of the assay (i.e., $6.2 \%$-equivalent to $5 \mu \mathrm{g}$ of aggregated gamma globulin).

\$IPF patients whose alveolar macrophages released large amounts of chemotactic factor (see Fig. 2).

" 9 of $10 \mathrm{IPF}+$ patients had increased Clq binding activity in lavage fluid. The mean Clq binding activity of lavage fluid in these patients is equivalent to $\sim 8 \mu \mathrm{g}$ of aggregated gamma globulin. It is unlikely that the $\mathrm{Clq}$ binding activity in the IPF + patients was due to the presence of aggregated IgG since there were similar amounts of $\operatorname{IgG}$ in lavage fluids of the IPF + patients compared to the IPF - patients $(P>0.2)$. I IPF patients whose alveolar macrophages released either no detectable amounts of the chemotactic factor or only small amounts of the factor (see Fig. 2).

All data is expressed as mean \pm SEM.

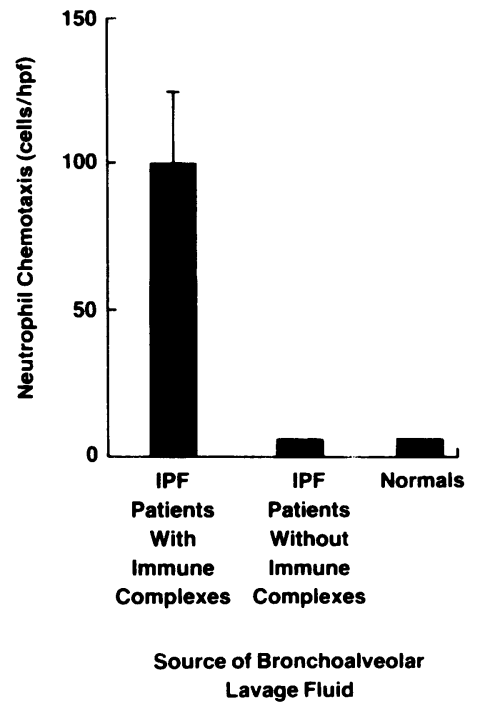

FigUre 5 Release of a chemotactic factor for neutrophils by alveolar macrophages from normal individuals following stimulation with bronchoalveolar lavage fluid from normal individuals and patients with IPF. The chemotactic activity of the macrophage supernates is on the ordinate (expressed as cells migrated/hpf) and the source of the lavage fluid is on the abscissa. The lavage fluids from patients with IPF are divided into two groups: lavage fluids from patients with circulating immune complexes and lavage fluids from patients without circulating immune complexes. No significant amounts of chemotactic activity were detected in supernates of macrophages exposed to bronchoalveolar lavage fluid from normal individuals or IPF patients without immune complexes. Sufficient amounts of chemotactic activity were present in undiluted supernates of macrophages exposed to bronchoalveolar lavage fluid from IPF patients with immune complexes to result in at least a $50 \%$ maximal migration of neutrophils in the chemotaxis assay.

the factor; for each of the groups studied, the lavage fluid itself was not chemotactic for neutrophils (data not shown). The failure of lavage fluid from normals or patients without circulating immune complexes to stimulate normal macrophages to release the factor was not due to the presence of decreased amounts of IgG in these lavage fluids; when the lavage fluids from both groups were adjusted to have an amount of IgG comparable to that present in lavage fluid from IPF patients with circulating immune complexes, the fluids still did not stimulate normal macrophages to release the chemotactic factor (data not shown).

Third, IPF alveolar macrophages which released large amounts of chemotactic factor exhibited an apparent suppression of $\operatorname{IgG} \mathrm{Fc}$ receptor function, suggesting their $F_{c}$ receptors were occupied (i.e., by IgG immune complexes). The release of the chemotactic factor by alveolar macrophages following in vitro stimulation with IgG-coated ORBC requires an available IgG Fc receptor, whereas Sepharose 4B stimulates macrophages by a mechanism independent of the $\mathrm{IgG}$ 
Fc receptor (20). Alveolar macrophages from both normals and IPF patients without circulating immune complexes released large amounts of chemotactic activity following in vitro stimulation with either IgGcoated ORBC or Sepharose 4B (Fig. 6). In contrast, the amount of chemotactic factor released by alveolar macrophages from IPF patients with circulating immune complexes was not increased following in vitro stimulation with IgG-coated ORBC $(P>0.2)$, but was increased following stimulation with Sepharose $4 \mathrm{~B}$ $(P<0.01)$.

Fourth, all IPF alveolar macrophages which released a chemotactic factor for neutrophils demonstrated intracytoplasmic granular fluorescence for IgG; only one of five of the IPF alveolar macrophages not releasing the factor and one of eight of the normal macrophage suspensions showed a similar pattern of fluorescence.

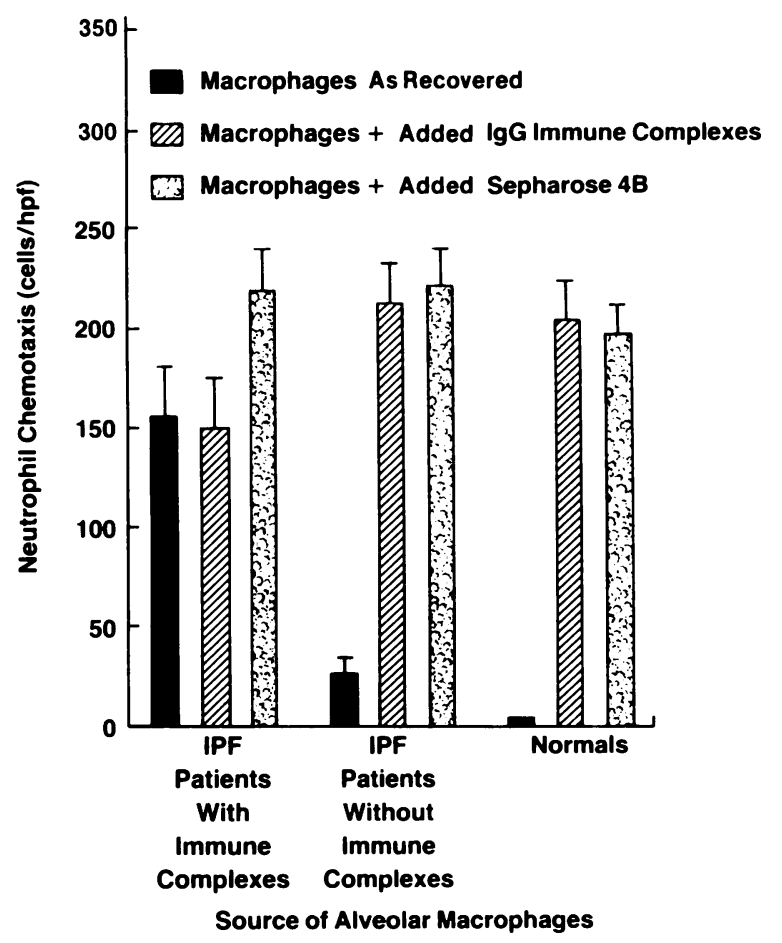

FIGURE 6 Role of immune complexes in causing release of a chemotactic factor by alveolar macrophages from patients with IPF. All macrophage cultures were for $3 \mathrm{~h}$. In some of the macrophage cultures no stimuli were added; in the other cultures, macrophages were stimulated with either IgC immune complexes (IgG-coated ORBC) and/or Sepharose 4B. The chemotactic activity of the macrophage cultures is on the ordinate (expressed as neutrophils migrated/ hpf) and the source of the alveolar macrophages is on the abscissa. The macrophages from patients with IPF are divided into two groups: macrophages from patients with circulating immune complexes and macrophages from patients without circulating immune complexes.
Further evidence suggesting IPF macrophages have occupied $\mathrm{Fc}_{\mathrm{c}}$ receptors came from studies demonstrating that alveolar macrophages from IPF patients with circulating immune complexes exhibited a defective killing of IgG-coated ORBC (Fig. 7). Alveolar macrophages kill IgG-coated ORBC via a mechanism that is mediated by the macrophage $\mathrm{IgG} F \mathrm{~F}$ receptor; $\mathrm{PHA}$ coated ORBC are killed by a mechanism that is independent of the macrophage $\operatorname{IgG} \mathrm{Fc}_{\mathrm{C}}$ receptor $(28,29)$. At various effector to target cell ratios, the killing of both IgG- and PHA-coated ORBC by alveolar macrophages was similar in normals and IPF patients without circulating immune complexes $(P<0.2$, each comparison). By comparison, the killing of IgG-coated ORBC by alveolar macrophages from IPF patients with circulating immune complexes was significantly decreased at each effector to target cell ratio compared with macrophages from both normals and IPF patients without circulating immune complexes $(P<0.01$, each comparison). The killing of PHA-coated ORBC by alveolar macrophages from IPF patients with circulating immune complexes was not significantly different from that of macrophages from normals or IPF patients without circulating immune complexes $(P>0.2$, each comparison).

\section{DISCUSSION}

The current concepts of the pathogenesis of IPF hold that destruction of the lung parenchyma results from the chronic presence of neutrophils in the alveolar structures $(5,10-16)$. It is likely that neutrophils mediate this parenchymal injury by secreting various enzymes, including collagenase, which are capable of deranging the connective tissue matrix of the lung (33-35). In addition, neutrophils that are present within the alveolar structures of patients with IPF may also directly injure lung parenchymal cells (36). Thus, since neutrophils are normally not present within the lung (11-16), at least one determinant of lung parenchymal injury in IPF is the mechanism(s) by which neutrophils are attracted to the alveolar structures.

The present study has delineated a mechanism by which neutrophils may be attracted to the lung in this disease: alveolar macrophages of patients with large numbers of neutrophils in their alveolar structures release a potent chemotactic factor for neutrophils. By comparison, alveolar macrophages of IPF patients with small numbers of neutrophils in their alveolar structures release only small amounts of the chemotactic factor. Thus, in patients with IPF, there appears to be an in vivo correlation between the numbers of neutrophils in the alveolar structures and the amounts of chemotactic factor that is released by alveolar macrophages. 


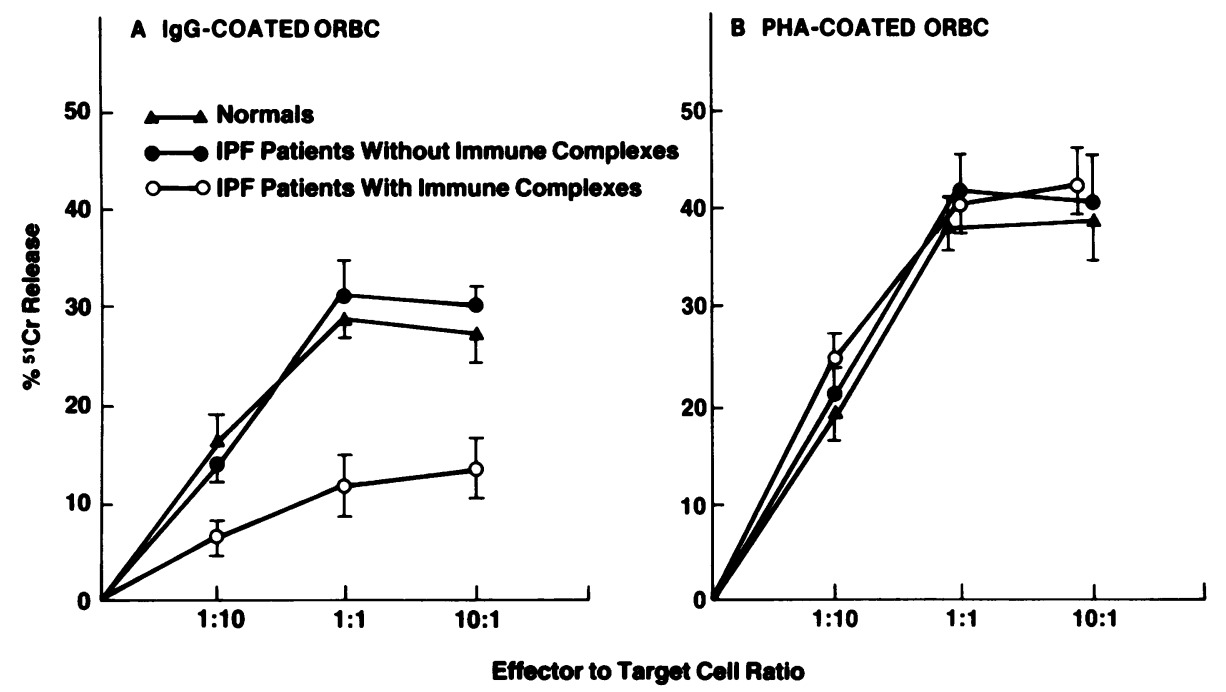

FIgURE 7 Comparison of the killing of IgG-coated ORBC and PHA-coated ORBC by alveolar macrophages from normal individuals and patients with IPF. The ORBC were labeled with ${ }^{51} \mathrm{Cr}$ and cell killing is expressed as percent ${ }^{51} \mathrm{Cr}$ release. The macrophages from patients with IPF are divided into two groups: macrophages from patients with circulating immune complexes and macrophages from patients without circulating immune complexes. The killing of the ${ }^{51} \mathrm{Cr}$ labeled ORBC is quantitated on the ordinate as the percent ${ }^{51} \mathrm{Cr}$ release and the ratio of effector cells (alveolar macrophages) to target cells (ORBC) is on the abscissa.

The chemotactic factor released by IPF alveolar macrophages is similar to the factor released by normal alveolar macrophages following in vitro stimulation with a variety of agents (20). It is of low molecular weight (400-600 mol wt), at least partially lipid in nature, and preferentially attracts neutriphils compared to monocytes.

Several lines of evidence suggest that immune complexes are the in vivo stimulus that induces the IPF alveolar macrophage to release the chemotactic factor for neutrophils.

(a) Immune complexes stimulate macrophages from normal individuals to release the chemotactic factor $(20)$.

(b) IPF patients whose macrophages release large amounts of the chemotactic factor have immune complexes in their bronchoalveolar lavage fluid. In contrast, lavage fluid from IPF patients whose alveolar macrophages release only small amounts of the factor do not contain detectable levels of immune complexes. These observations are consistent with previous studies that have also demonstrated immune complexes in serum and in lung biopsies of patients with IPF that have intense alveolitis (37).

(c) In patients with IPF, only bronchoalveolar lavage fluid with elevated levels of immune complexes stimulates normal macrophages to release the chemotactic factor.

(d) All alveolar macrophages which released the chemotactic factor for neutrophils showed intracytoplasmic granular fluorescence for IgG, suggesting that IgG immune complexes had been ingested by these cells.

(e) In untreated patients with IPF, alveolar macrophages that release large amounts of the chemotactic factor exhibit decreased function of their IgG Fc receptor. In contrast, the IgG Fc receptor function of IPF macrophages that release only small amounts of the factor is similar to that of normal macrophages. The apparent altered function of the Fc receptor of macrophages that released the chemotactic factor appears not to be a generalized inhibition of macrophage function since these cells function normally when stimulated by mechanisms that are independent of the IgG Fc receptor. A likely mechanism for the altered Fc receptor function of these cells is that immune complexes are bound to the macrophage Fc receptor. Similar alterations in $\mathrm{Fc}$ receptor function are found on macrophages of the spleen and liver in other types of patients with circulating immune complexes $(38,39)$. In addition, Griffin has also demonstrated that following ingestion of immune complexes, macrophages exhibit a selective alteration of their IgG Fc receptor function (40).

In addition to immune complexes, other agents such as microorganisms and inorganic particulates are known to stimulate alveolar macrophages to release the chemotactic factor for neutrophils $(19,20,22)$. It is unlikely that IPF alveolar macrophages release the chemotactic factor as a result of an interaction with microorganisms since lung biopsies of these patients, 
bronchoalveolar lavage fluid, and the macrophage cultures themselves were sterile. In addition, none of the patients had a history of exposure to inorganic materials known to cause interstitial lung disease and these materials were not found on direct analysis of the lung biopsies. In addition to these types of inorganic particulates, the particulates that are present in cigarette smoke also stimulate alveolar macrophages to release a chemotactic factor for neutrophils (23). However, the IPF patients in the present study either were nonsmokers or had discontinued smoking for at least $1 \mathrm{yr}$. Therefore, it is unlikely that the release of the chemotactic factor by alveolar macrophages from these patients can be attributed to the presence of particulates of cigarette smoke in the lower respiratory tract.

Although it is clear that alveolar macrophages of some IPF patients release large amounts of a potent chemotactic factor for neutrophils, it is possible that other chemotactic factors, such as the complementderived chemotactic factors, are also generated as a result of the presence of immune complexes in lung as well as in blood of patients with this disorder (41-45). It is unlikely, however, that the complement-derived chemotactic factors that are generated in the vascular spaces are important in terms of the pathogenesis of IPF since experimental studies have demonstrated that, in the vascular spaces, these factors do not mediate migration of neutrophils into the alveolar structures (46). In support of this concept are studies showing that patients with active systemic lupus erythematosis (SLE) have only small numbers of neutrophils in the lung in spite of the fact that these patients have high titers of circulating immune complexes and activation of the complement system (12). In addition, alveolar macrophages of patients with active SLE either do not release detectable amounts of the chemotactic factor or release only small amounts of the factor (data not shown). Since both patients with active SLE and active IPF have circulating immune complexes $(37,38)$, these observations also suggest that either not all circulating immune complexes reach the surface of the alveolar macrophage, or that certain immune complexes that are present in the lung do not stimulate macrophages to release the chemotactic factor. Alternatively, it is reasonable to hypothesize that, in IPF, the immune complexes are formed in lung and are subsequently spilled over into blood. The latter possibility is attractive in terms of the pathogenesis of IPF since this disorder, in contrast to SLE, is not a systemic disorder but is limited to lung. In support of this hypothesis are studies that demonstrate that, in IPF, IgG production (and, thus, likely also immune complex formation) is localized to lung $(47-48)$ whereas in SLE increased amounts of IgG are also produced in extrapulmonary sites (48).
Since complement proteins are present in the alveolar structures of patients with IPF $(12,25)$, it is possible that chemotactic factors could be generated from these proteins following an interaction with immune complexes. However, even if these complement-derived chemotactic factors are generated in the lung in IPF, it is possible that the primary effect of these factors on neutrophil migration to the lung is mediated via stimulation of alveolar macrophages to release the macrophage-derived chemotactic factor (21-22). In support of this hypothesis are recent studies by Henson et al. (21) which demonstrated that, following intratracheal injection of a purified complement-derived chemotactic factor $\left({\mathrm{C} 5 \mathrm{a}_{\text {des }} \mathrm{Arg}}\right)$ into rabbit lung, the neutrophils which were attracted to the lung exhibited a greater "desensitization" to the macrophage-derived factor than to the complement-derived factor (21). These neutrophils were "desensitized" to the macrophagederived factor, presumably by prior in vivo exposure to the macrophage-derived chemotactic factor. However, independent of whether neutrophils are attracted to the lung in IPF solely by the macrophagederived chemotactic factor or by this factor in conjunction with other chemotactic factors, it is clear that the alveolar macrophage plays a central role in modulating the migration of neutrophils to the lung by secreting a potent chemotactic factor for neutrophils.

\section{REFERENCES}

1. Spencer, H. 1967. Interstitial pneumonia. Annu. Rer. Med. 18: 423-442.

2. Scadding, J. G., and K. F. W. Hinson. 1967. Diffuse fibrosing alveolitis (diffuse interstitial fibrosis of the lungs): correlation with histology at biopsy with prognosis. Thorax 22: 291-304.

3. Hinson, K. F. W. 1970. Diffuse pulmonary fibrosis. Hum. Pathol. 1: 275-288.

4. Scadding, J. G. 1974. Diffuse pulmonary alveolar fibrosis. Thorax 29: 271-281.

5. Crystal, R. G., J. D. Fulmer, W. C. Roberts, M. L. Moss, B. R. Line, and H. Y. Reynolds. 1976. Idiopathic pulmonary fibrosis: clinical, histologic, radiographic, physiologic, scintigraphic, cytologic, and biochemical aspects. Ann. Intern. Med. 85: 769-788.

6. Fulmer, J. D., and R. G. Crystal. 1976. The biochemical basis of pulmonary function. In The biochemical basis of pulmonary function. R. G. Crystal, editor. Marcel Dekker, New York. 419-466.

7. Weinberger, S. E., and R. G. Crystal. 1979. Reactions of the interstitial space to injury. In Textbook of Pulmonary Disease. A. P. Fishman, editor. McGraw-Hill, Inc. New York. 640-647.

8. Basset, F., P. Soler, and J. F. Bernandin. 1975. Contribution of electron microscopy to the study of interstitial pneumonias. Prog. Respir. Dis. 8: 45-58.

9. Brody, A. R., and J. E. Craighead. 1976. Interstitial association of cells lining air spaces in human pulmonary fibrosis. Virchows Arch. (Pathol. Anat.) 372: 3949. 
10. Fulmer, J. D., and R. G. Crystal. 1979. Interstitial lung disease. In Current Pneumonology. D. H. Simmons, editor. Houghton Mifflin, Boston. p. 1-65.

11. Hunninghake, G. W., J. D. Fulmer, R. C. Young, J. E. Gadek, and R. G. Crystal. 1979. Localization of the immune response in sarcoidosis. Am. Rev. Respir. Dis. 120: 49-57.

12. Hunninghake, G. W., J. E. Gadek, O. Kawanami, V. J. Ferrans, and R. G. Crystal. 1979. Inflammatory and immune processes in the human lung in health and disease: evaluation by bronchoalveolar lavage. Am. J. Pathol. 97: 149-206.

13. Crystal, R. G., V. J. Ferrans, J. E. Gadek, J. D. Fulmer, B. R. Line, and G. W. Hunninghake. 1981. Interstitial lung disease: current concepts of pathogenesis, staging, and therapy. Am. J. Med. 70: 542-568.

14. Weinberger, S. E., J. A. Kelman, N. A. Elson, R. C. Young, H. Y. Reynolds, J. D. Fulmer, and R. G. Crystal. 1978. Bronchoalveolar lavage in interstitial lung disease. Ann. Intern. Med. 89: 459-466.

15. Hunninghake, G. W., J. E. Gadek, S. E. Weinberger, J. A. Kelman, N. A. Elson, R. C. Young, J. D. Fulmer, and R. G. Crystal. 1979. Comparison of the alveolitis of sarcoidosis and idiopathic pulmonary fibrosis. Chest. 75(Suppl): 266-267.

16. Hunninghake, G. W., O. Kawanami, V. J. Ferrans, W. C. Roberts, and R. G. Crystal. 1981. Characterization of the inflammatory and immune effector cells in the lung parenchyma of patients with interstitial lung disease. Am. Rev. Respir. Dis. 123: 407-412.

17. Kazmierowski, J. A., J. I. Gallin, and H. Y. Reynolds. 1977. Mechanism for the inflammatory response in primate lungs. Demonstration and partial characterization of an alveolar macrophage-derived chemotactic factor with preferential activity for polymorphonuclear leukocytes. J. Clin. Invest. 59: 273-281.

18. Hunninghake, G. W., J. I. Gallin, and A. S. Fauci. 1978. Immunological reactivity of the lung. The in vivo and in vitro generation of a neutrophil chemotactic factor by alveolar macrophages. Am. Rev. Respir. Dis. 117: 15-23.

19. Merrill, W. W., G. P. Naegel, R. A. Matthay, and H. Y. Reynolds. 1980. Alveolar macrophage-derived chemotactic factor. Kinetics of in vitro production and partial characterization. J. Clin. Invest. 65: 268-276.

20. Hunninghake, G. W., J. E. Gadek, H. Fales, and R. G. Crystal. 1980. Human alveolar macrophage-derived chemotactic factor for neutrophils: stimuli and partial characterization. J. Clin. Invest. 66: 473-483.

21. Henson, P. M., K. McCarthy, G. L. Larsen, R. O. Webster, P. C. Giclas, R. B. Dreisin, T. E. King, and J. O. Shaw. 1979. Complement fragments, alveolar macrophages, and alveolitis. Am. J. Pathol. 97: 93-110.

22. Gadek, J. E., G. W. Hunninghake, R. L. Zimmerman, and R. G. Crystal. 1980. Regulation of release of alveolar macrophage-derived neutrophil chemotactic factor. Am. Rev. Respir. Dis. 121: 723-733.

23. Hunninghake, G. W., S. V. Szapiel, and R. G. Crystal. 1980. Mechanisms by which cigarette smoke attracts polymorphonuclear leukocytes to lung. Chest. 77(Suppl.): 373.

24. Böyum, A. 1968. Isolation of mononuclear cells and granulocytes from human blood. Scand. J. Clin. Invest. 21(Suppl. 97): 77-89.

25. Reynolds, H. Y., J. D. Fulmer, J. A. Kazmierowski, W. C. Roberts, M. M. Frank, and R. G. Crystal. 1977. Analysis of cellular and protein content of bronchoalveolar lavage fluid from patients with idiopathic pul- monary fibrosis and chronic hypersensitivity pneumonitis. J. Clin. Invest. 59: 165-175.

26. Zubler, R. H., G. Lange, P. H. Lambert, and P. A Miescher. 1976. Detection of immune complexes in unheated sera by a modified ${ }^{125} \mathrm{I}-\mathrm{Clq}$ binding test. $\mathrm{J}$. Immunol. 116: 232-240.

27. Lawley, T. J., H. M. Moutsopoulos, S. I. Katz, A. N Theofilopoulos, T. M. Chused, and M. M. Frank. 1979. Demonstration of circulating immune complexes in Sjögrens syndrome. J. Immunol. 123: 1382-1387.

28. Hunninghake, G. W., and A. S. Fauci. 1977. Immunologic reactivity of the lung. III. Effects of corticosteroids on alveolar macrophage cytotoxic effector cell function. J. Immunol. 118: 146-150.

29. Hunninghake, G. W., and A. S. Fauci. 1977. Immunological reactivity of the lung. VII. Effect of corticosteroids and cyclophosphamide on the $F_{c}$ receptor function of alveolar macrophages. Cell. Immunol. 32: 228-233.

30. Hunninghake, G. W., J. E. Gadek, S. V. Szapiel, I. J. Strumpf, O. Kawanami, V. J. Ferrans, and R. G. Crystal. 1980. The human alveloar macrophage. In Methods in Cell Biology. Vol. 21A, C. C. Harris, B. F. Trump, and G. D. Stoner, editors. Academic Press, Inc., New York. pp. 95-112.

31. Hunninghake, G. W., and A. S. Fauci. 1976. Divergent effects of cyclophosphamide administration on mononuclear killer cells: quantitative depletion of cell numbers versus qualitiative suppression of functional capabilities. J. Immunol. 117: 337-342.

32. Hunninghake, G. W., and A. S. Fauci. 1977. Lymphocytemediated cytotoxicity against human allogeneic and autologous lymphoid targets after concanavalin-A activation of cytotoxic effector cells. J. Immunol. 119: 1122-1128.

33. Horwitz, A. L., A. J. Hance, and R. G. Crystal. 1977. Granulocyte collagenase: selective digestion of type I relative to type III collagen. Proc. Natl. Acad. Sci. U. S. A. 74: 897-901.

34. Hance, A. J., and R. G. Crystal. 1975. The connective tissue of the lung. Am. Rev. Respir. Dis. 112: 657-711.

35. Gadek, J. E., J. A. Kelman, S. E. Weinberger, A. L. Horwitz, H. Y. Reynolds, J. D. Fulmer, and R. G. Crystal. 1979. Collagenase in the lower respiratory tract of patients with idiopathic pulmonary fibrosis. N. Engl.J. Med. 301: 737-742.

36. Hunninghake, G. W., S. V. Szapiel, J. D. Fulmer, B. Keogh, and R. G. Crystal. 1979. Neutrophil-mediated fibroblast destruction in idiopathic pulmonary fibrosis (IPF). Am. Rev. Respir. Dis. 119: 72A.

37. Dreisin, R. B., M. I. Schwartz, A. N. Theofilopoulos, and R. E. Stanford. 1978. Circulating immune complexes in the idiopathic interstitial pneumonias. $N$. Engl. J. Med. 298: 353-357.

38. Frank, M. M., M. I. Hamburger, T. J. Lawley, R. P. Kimberly, and P. H. Plotz. 1979. Defective reticuloendothelial system Fc-receptor function in systemic lupus erythematosis. N. Engl. J. Med. 300: 518-523.

39. Jaffe, C. J., J. M. Vierling, E. A. Jones, T. J. Lawley, and M. M. Frank. 1978. Receptor specific clearance by the reticuloendothelial system in chronic liver diseases. Demonstration of defective C3b-specific clearance in primary biliary chirrosis. J. Clin. Invest. 62: 1069-1077.

40. Griffin, F. M. 1980. Effects of soluble immune complexes on $F_{c}$ receptor and $\mathrm{C} 3 \mathrm{~b}$ receptor mediated phagocytosis by macrophages. J. Exp. Med. 152: 905-919.

41. Gallin, J. I., R. A. Clark, and M. M. Frank. 1975. Kinetic analysis of chemotactic factor generation in human serum 
via activation of the classical and alternate complement pathways. Clin. Immunol. Immunopathol. 3: 334-346.

42. Ward, P. A., G. G. Cochrane, and J. H. Muller-Eberhard. 1965. The role of serum complement in chemotaxis of leukocytes in vitro. J. Exp. Med. 122: 327-346.

43. Snyderman, R., H. Gewarz, and S. E. Mergenhagen. 1968. Interactions of the complement system with endotoxin lipopolysaccharide. J. Exp. Med. 128: 259-275.

44. Snyderman, R., H. S. Shin, J. K. Phillips, H. Gewurz, and S. E. Mergenhagen. 1969. A polymorphonuclear leukocyte chemotactic activity in rabbit and guinea pig serum treated with immune complexes. Evidence for $\mathrm{C} 5 \mathrm{a}$ as the major chemotactic factor. Infect. Immun. 1: $521-525$.

45. Fernandez, H. N., P. M. Henson, A. Otani, and T. E.
Hugli. 1978. Chemotactic response to human C3a and C5a anaphylatoxins. Evaluation of C3a and C5a leukotaxis in vitro and under simulated in vivo conditions. J. Immunol. 120: 109-114.

46. Ward, P. A. 1979. Immune complex injury to the lung. Am. J. Pathol. 97: 85-92.

47. Lawrence, E. C., R. R. Martin, R. M. Blaese, R. B. Teagil, R. J. Awe, R. K. Wilson, W. J. Deaton, K. Bloom, S. D. Greenberg, P. M. Stevens. 1980. Increased bronchoalveolar IgG-secreting cells in interstitial lung disease. N. Engl. J. Med. 302: 1186-1188.

48. Hunninghake, G. W., and R. G. Crystal. Localization of immunoglobulin production to sites of disease activity in idiopathic pulmonary fibrosis. Am. Rev. Respir. Dis. In press. 